\title{
Über Chlor-m-Phenylendiamin
}

von

\section{Dr. Paul Cohn und Dr. Armin Fischer.}

Aus dem chemischen Laboratorium des Prof. Dr. Ed. Lippmann an der k. k. Universität in Wien.

(Vorgelegt in der Sitzung am 8. Februar 1900.)

Von den drei theoretisch vom $m$-Phenylendiamin ableitbaren Monochlorsubstitutionsproducten ist nur ein einziges in der Literatur beschrieben. Es ist dies das 4-Chlor-m-Phenylendiamin, über welches Beilstein und Kurbatow nur sehr dürftige Angaben machen. Dieselben haben das 4-Chlor-m-Phenylendiamin durch Reduction von 4-Chlor-m-Dinitrobenzol mit Zinn und Salzsäure erhalten und beschreiben es als einen bei $86^{\circ}$ schmelzenden Körper, der aus Ligroin krystallisiert und in Alkohol und Chloroform löslich ist. Es ist auch nur eine einzige Chlorbestimmung der Substanz angeführt.

Wie hieraus zu ersehen ist, bedurften diese Angaben einer wesentlichen Ergänzung, da weder Substitutionsproducte, noch Salze beschrieben sind. Während unsere Untersuchung im Gange war, entnahmen wir einer Mittheilung der Chemikerzeitung, ${ }^{2}$ dass der Actiengesellschaft für Anilinfabrication in Berlin die Darstellung eines neuen symmetrischen Chlor$m$-Phenylendiamins $(1-3-5)$ vom Schmelzpunkte $103^{\circ}$ gelungen sei, und dass dieser Körper unter dem Namen »Nerogen $D$ « in den Handel gebracht werde, um als Entwickler für Sambesischwarz- $D$-Färbungen Anwendung zu finden. Durch diese Mittheilung angeregt, beschäftigten wir uns mit dem Nerogen $D$ und erkannten, dass diesem Producte nicht die im

\footnotetext{
1 Ann., 197, S. 76.

2 Jahrg. XXIII, S. 1085 (30. December 1899).
} 
französischen Patente Nr. 286.888 vom 16. März 1899 angegebene symmetrische Constitution zukomme, sondern dass es mit dem von uns bearbeiteten 4-Chlor-m-Phenylendiamin identisch sei, was auch die krystallographische Untersuchung, die Herr Hofrath Prof. Lang vorzunehmen die Freundlichkeit hatte, bestätigte.

Den Angaben der Fabrik zufolge sollte das neue symmetrische Chlor-m-Phenylendiamin durch Reduction des schon von Bader ${ }^{1}$ auf anderem Wege erhaltenen und nur kurz beschriebenen 1-3-5-Dinitrochlorbenzols zu erhalten sein, welcher Körper nach jener Mittheilung durch Behandlung von $m$-Dinitrobenzol bei Gegenwart eines Chlorüberträgers mit gasförmigem Chlor und Einhaltung der Temperatur von $95^{\circ}$ bis $100^{\circ}$ gewonnen werden sollte. Es gelang uns jedoch nicht, diese Angaben zu bestätigen, obwohl wir mehrere Chlorüberträger zur Anwendung brachten. So führten Chlorierungsversuche von tagelanger Dauer mit absolut trockenem Chlor unter Verwendung von Eisendraht, von Eisenpulver und von Aluminiumchlorid als Überträger $z u$ keinem Resultate; es gelang nicht, aus der Reactionsmasse, die übrigens selbst bei tagelangem Einleiten nur wenig Chlor aufnahm und zum allergrößten Theile unverändertes $m$-Dinitrobenzol enthielt, das gewünschte Chlor-m-Dinitrobenzol zu erhalten. Die Arbeit wird nach dieser Richtung fortgesetzt.

\section{Darstellung von 4-Ch1or-m-Phenylendiamin.}

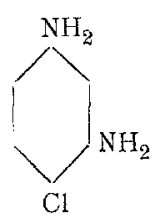

Bei der Darstellung der Base verfuhren wir folgendermaßen:

$9 g$ 4-Chlor-(1-3)-Dinitrobenzol wurden in äußerst kleinen Portionen in eine salzsaure Lösung (conc. $\mathrm{HCl}$ ) von $20 \mathrm{~g}$ krystallisiertem Zinnchlorür eingetragen und etwa 2 Minuten

1 Berl. Ber., XXIV, 1665 ff. (1891). 
lang erwärmt, bis ein $\ddot{O l}$ an der Flüssigkeitsoberfäche erschien. Es wurde hierauf kräftig umgeschüttelt, so lange bis das Ö1 im Reactionsgemische verschwunden war. Eine eventuell zu große Temperatursteigerung musste man durch Wasserkühlung vermeiden. Es wurde nach Beendigung der Reduction mit der acht- bis zehnfachen Wassermenge verdünnt, das Zinn durch Einleiten von Schwefelwasserstoff ausgefällt, hierauf das Schwefelzinn durch Filtration entfernt und die Base mit Natronlauge frei gemacht. Durch Schütteln mit Äther wurde das Chlorphenylendiamin aus dem Gemische aufgenommen, der Äther wieder abdestilliert und die zurückbleibende Base aus Chloroform umkrystallisiert. Das so dargestellte 4-Chlor-m-Phenylendiamin krystallisiert in schönen, wohlausgebildeten Krystallen.

Herr Hofrath Prof. Lang hatte die Güte, eine Messung derselben vorzunehmen und theilt uns hierüber Folgendes mit: "An den in das rhombische System gehörenden Krystallen herrschen die Flächen der Zone [001] vor, in welchen die Formen (110) und (100) vorkommen. Die letztere Fläche ist nun entweder sehr stark entwickelt, und dann haben die Krystalle einen plattenförmigen Habitus, oder sie tritt ganz untergeordnet auf, in welchem Falle die Krystalle lange Nadein bilden, die sich nicht zur Messung eignen. Die plattenförmigen Krystalle, welche noch die Form (110) zeigen, konnten dagegen mit genügender Genauigkeit gemessen werden; ihr Axenverhältnis ist:

$$
a: b: c=1: 0 \cdot 7106: 0 \cdot 6375 .
$$

Die Ergebnisse der optischen Untersuchung sprechen ebenfalls für das rhombische System «.

Die Base ist in vollkommen reinem Zustande farblos, hat aber Neigung, sich röthlich zu färben; diese Färbung rührt von der Oxydation her, welche sie an der Luft erleidet. In vollkommen trockenem Zustande hält sich die Substanz lange unverändert. Der Körper ist schwer löslich in kaltem, viel leichter in heißem Wasser, aus welchem er gut krystallisiert. In Alkohol ist das Chlorphenylendiamin sehr leicht löslich, etwas schwerer in Äther, noch schwerer in Benzol. Die schönsten Krystalle der Substanz sind aus heißer Chloroformlösung zu 
erhalten. Den Schmelzpunkt der Base fanden wir trotz vielfacher Umkrystallisierung aus verschiedenen Lösungsmitteln bei $91^{\circ}$, während Beilstein und Kurbatow ihn bei $86^{\circ}$ angeben. Zum Zwecke der genauen Feststellung des Schmelzpunktes wurde analysiertes 4-Chlor- $m$-Phenylendiaminoxalat mittels Kalilauge zerlegt und der Schmelzpunkt der so frei gewordenen Base nach mehrfachem Umkrystallisieren aus Chloroform bestimmt, und liegt derselbe gleichfalls bei $91^{\circ}$.

Die heiße wässerige Lösung reduciert Silbernitratiösung. Versetzt man die heiße, wässerige, angesäuerte Lösung des 4-Chlor-m-Phenylendiamins mit einem Tropfen Eisenchlorid, so erfolgt rasche Oxydation, und die farblose Lösung wird orangeroth, kirschroth, dann dunkelroth. Dieselbe Erscheinung erfolgt bei Zusatz eines Tropfens Kaliumbichromat zur angesäuerten, erwärmten wässerigen Lösung.

Analyse:

I. $0 \cdot 1605 \mathrm{~g}$ Substanz ergaben, mit Kupferoxydasbest verbrannt, $0.2960 \mathrm{~g}$ Kóhlensäure und $0.0740 \mathrm{~g}$ Wasser.

II. $0 \cdot 2490 \mathrm{~g}$ Substanz ergaben bei der Chlorbestimmung nach Carius $0 \cdot 2480 \mathrm{~g}$ Chlorsilber.

In 100 Theilen:

\begin{tabular}{|c|c|c|}
\hline & $\begin{array}{c}\text { Berechnet für } \\
\mathrm{C}_{6} \mathrm{H}_{7} \mathrm{~N}_{2} \mathrm{Cl}\end{array}$ & Gefunden \\
\hline C. & $50 \cdot 25$ & $50 \cdot 28$ \\
\hline $\mathrm{H} \ldots \ldots$ & $4 \cdot 91$ & $5 \cdot 12$ \\
\hline $\mathrm{Cl} \ldots . .$. & $24 \cdot 91$ & $24 \cdot 60$ \\
\hline
\end{tabular}

Chlorhydrat. $\mathrm{C}_{6} \mathrm{H}_{3} \leq \begin{aligned} & \mathrm{NH}_{2} \\ & \mathrm{NH}_{2} \\ & \mathrm{Cl} .\end{aligned}$

In die ätherische Lösung von 4-Chlor-m-Phenylendiamin wurde trockene Salzsäure eingeleitet. Es fiel ein weißer seidenglänzender Niederschlag, der, mit Alkohol und Äther gut nachgewaschen, im Vacuumexsiccator getrocknet wurde.

Eigenschaften. Das Chlorhydrat bildet weiße feine Nädelchen, die in Wasser leicht löslich sind. Geringer ist die 
Löslichkeit in Alkohol. In Äther ist die Substanz unlöslich. Erhitzt man dieselbe, so tritt gegen $205^{\circ}$ Zersetzung unter Schwarzfärbung ein. Die wässerige erwärmte Lösung färbt sich bei Zusatz eines Tropfens Eisenchlorid oder Kaliumbichromat erst orange, dann immer tiefer roth.

Analyse:

0.1815 g Substanz ergaben nach Carius 0.2930 g Chlorsiber.

In 100 Theilen:

$$
\begin{aligned}
& \text { Berechnet für } \\
& \mathrm{Cl} \ldots \ldots \underbrace{\mathrm{C}_{6} \mathrm{H}_{8} \mathrm{~N}_{2} \mathrm{Cl}_{2}}_{39 \cdot 58} \quad \underbrace{\text { Gefunden }}_{39 \cdot 78} \\
& \text { Platindoppelsalz. } \\
& 2\left[\mathrm{C}_{6} \mathrm{H}_{3}\left(\mathrm{NH}_{2}\right)_{2} \mathrm{Cl} \text {. } \mathrm{HCl}\right]+\mathrm{PtCl}_{4} \text {. }
\end{aligned}
$$

Man versetzt die concentrierte wässerige Lösung des salzsauren 4-Chlor-m-Phenylendiamins mit concentrierter wässeriger Platinchloridlösung. Es fällt nach längerem Reiben mit dem Glasstab das Platindoppelsalz in kleinen, glänzenden, gelb gefärbten Krystallen aus, die abgesaugt und gut mit Alkohol und Alkohol-Äther gewaschen werden.

Analyse:

$0 \cdot 3720 \mathrm{~g}$ Substanz gaben $0 \cdot 1051 \mathrm{~g}$ Platin.

In 100 Theilen:

$$
\text { Pt....... } \underbrace{\begin{array}{c}
\text { Berechnet für } \\
\mathrm{C}_{12} \mathrm{H}_{16} \mathrm{~N}_{4} \mathrm{Cl}_{8} \mathrm{Pt}
\end{array}}_{28.08} \quad \underbrace{\text { Gefunden }}_{28 \cdot 23}
$$

Sulfat.

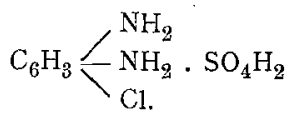

$5 g$-Chlor- $m$-Phenylendiamin wurden in Äther gelöst und hierauf eine Lösung von $3 \cdot 5 \mathrm{~g}$ concentrierter Schwefelsäure in Äther allmählich zugefügt. Es fiel in weißen Massen das Sulfat aus, das gut mit Äther gewaschen wurde. Aus heißem Wasser ließ sich das Salz in sehr schönen, weißen, manchmal schwach röthlichen Nadeln umkrystallisieren. 
Eigenschaften. Schöne farblose Nadeln, leicht löslich in heißem Wasser, viel schwerer in kaltem, in Alkohol sehr schwer löslich. Beim Erhitzen erfolgt gegen $155^{\circ}$ Zersetzung, mit Eisenchlorid oder Kaliumbichromat die bereits beschriebene Rothfärbung, welche Reaction allen Salzen des Chlorphenylendiamins zukommt.

Vom Sulfat wurde eine Schwefelbestimmung nach Carius durchgeführt.

Analyse:

$0.1710 \mathrm{~g}$ Substanz gaben $0.1635 \mathrm{~g}$ Bariumsulfat.

In 100 Theilen:

$$
\text { S . . . } \underbrace{\begin{array}{c}
\text { Berechnet für } \\
\mathrm{C}_{6} \mathrm{H}_{9} \mathrm{~N}_{2} \mathrm{O}_{4} \mathrm{ClS}
\end{array}}_{13 \cdot 30} \quad \underbrace{\text { Gefunden }}_{13 \cdot 19}
$$

Oxalat.

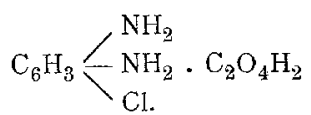

Man vereinigte die möglichst concentrierten alkoholischen Lösungen von $3 \mathrm{~g}$ Chlorphenylendiamin und $2.6 \mathrm{~g}$ krystallisierter Oxalsäure. Es fiel das Oxalat in weißen Massen aus, die abfiltriert, mit Alkohol gut nachgewaschen und aus heißem Alkohol umkrystallisiert wurden.

Eigenschaften. Weiße, seidenglänzende, feine Nadeln. Schwer löslich in kaltem Alkohol, leichter in warmem. Unlöslich in Äther und Benzol, leicht löslich in Wasser.

Erhitzt man das Oxalat, so erfolgt ge gen $185^{\circ}$ Zersetzung. Mit Eisenchlorid oder Kaliumbichromat geben die warmen, wässerigen Lösungen die schon oben beschriebene Rothfärbung.

Analyse:

$0.3340 \mathrm{~g}$ geben nach Carius, im Druckrohr erhitzt, $0 \cdot 2455 \mathrm{~g}$ Silberchlorid.

In 100 Theilen:

$$
\mathrm{Cl} \ldots \ldots \underbrace{\begin{array}{c}
\text { Berechnet für } \\
\mathrm{C}_{8} \mathrm{H}_{9} \mathrm{~N}_{2} \mathrm{O}_{4} \mathrm{Cl}
\end{array}}_{15 \cdot 27} \quad \underbrace{\text { Gefunden }}_{15 \cdot 16}
$$


Tartrat.

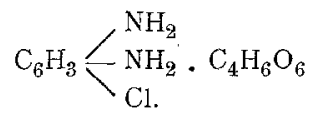

Es wurden die möglichst concentrierten Lösungen von $5 \mathrm{~g}$ Chlor-Phenylendiamin und $5.4 \mathrm{~g}$ Weinsäure in Alkohol vereinigt. Es fiel sofort das Tartrat als weißer voluminöser Niederschlag, der aus heißem Alkohol umkrystallisiert wurde.

Eigenschaften. Schöne weiße Nadeln, die Neigung zur Röthlichfärbung besitzen. Leicht löslich in Wasser, schwer in heißem Alkohol. In Äther und Benzol unlöslich. Mit Eisenchlorid oder Kaliumbichromat in wässeriger Lösung erwärmt, erfolgt Rothfärbung.

Analyse:

$0.2895 \mathrm{~g}$ Substanz gaben, nach Carius im Rohre erhitzt, $0 \cdot 1401 \mathrm{~g}$ Chlorsilber.

In 100 Theilen:

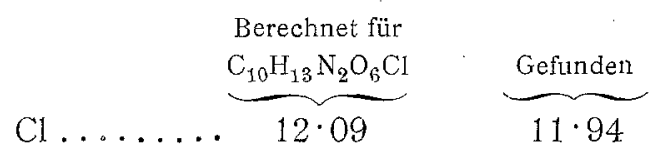

Monoacetyl-4-Chlor- $m$-Phenylendiamin.

$$
\mathrm{C}_{6} \mathrm{H}_{3} \leq \begin{aligned}
& \mathrm{NH} . \mathrm{CO} . \mathrm{CH}_{3} \\
& \mathrm{NH}_{2} \\
& \mathrm{Cl} .
\end{aligned}
$$

Bei der Acetylierung des Chlorphenylendiamins gelang es uns, ein einheitliches Acetylderivat nur nach der Methode von Hinsberg ${ }^{1} \mathrm{zu}$ gewinnen. $10 \mathrm{~g}$ Chlorphenylendiamin wurden, fein gepulvert, in einem Kölbchen mit etwas Wasser und Natronlauge versetzt und allmählich unter Eiskühlung und Schütteln gegen $16 \mathrm{~g}$ Acetanhydrid zugefügt. Zum Schlusse der Einwirkung reagierte das Reactionsgemisch noch alkalisch. Der abgeschiedene Acetylkörper wurde abfiltriert und mit Wasser gut gewaschen. Hierauf wurde er aus heißem Wasser

1 Berl. Ber., XXIII, 1890, S. 2962. 
unter Zusatz einer kleinen Menge Thierkohle behufs gänzlicher Entfärbung umkrystallisiert. Wie die Analyse ergab, hatten wir das Monoacetyl-4-Chlor- $m$-Phenylendiamin erhalten, obgleich genügend Anhydrid zur Bildung des Diacetylderivates bei der Darstellung vorhanden war.

Eigenschaften. Sehr schöne, weiße Nadeln, schwer löslich in kaltem Wasser, leicht in heißem, leicht löslich in Alkohol, schwer löslich in Benzol und Äther, ebenso in Chloroform, doch daraus in schönen Nadeln zu erhalten. Der Schmelzpunkt des Körpers liegt bei $170^{\circ}$. Mit Eisenchlorid oder Kaliumbichromat erfolgt in der erwärmten wässerigen Lösung nur nach dem Ansäuern erst Gelb-, dann Rothfärbung.

Analyse:

$0.2391 \mathrm{~g}$ Substanz geben, nach Carius behandelt, $0.1825 \mathrm{~g}$ Chlorsilber.

In 100 Theilen:

$$
\mathrm{Cl} \ldots \ldots \ldots \frac{\mathrm{C}_{8} \mathrm{H}_{9} \mathrm{~N}_{2} \mathrm{OCl}}{19 \cdot 17} \quad \underbrace{\text { Gefunden }}_{18 \cdot 86}
$$

\section{Dibenzoy1-4-Chlor- $m$-Phenylendiamin.}

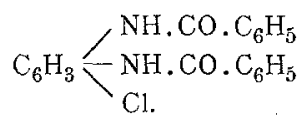

Die Benzoylierung erfolgte nach der Methode von Schotten-Baumann. $5 \mathrm{~g}$ Substanz wurden fein gepulvert, mit Wasser und Natronlauge versetzt und mit Benzoylchlorid geschüttelt. Hierauf wurde der Benzoylkörper abfiltriert, mit Wasser gut nachgewaschen, mit warmer verdünnter Essigsäure angerieben, um eventuell vorhandenes unverändertes Chlorphenylendiamin zu lösen, abfiltriert, getrocknet und aus Benzol umkrystallisiert.

Eigenschaften. Der so erhaltene Benzoylkörper krystallisiert in schönen weißen Nadeln vom Schmelzpunkte $178^{\circ}$. Er ist leicht löslich in Benzol, Alkohol, Chloroform, schwer in Äther, unlöslich in Wasser. 
Analyse:

$0.2801 \mathrm{~g}$ Substanz gaben nach Carius $0.1105 \mathrm{~g}$ Chlorsilber. In 100 Theilen:
Berechnet für
$\mathrm{C}_{20} \mathrm{H}_{15} \mathrm{~N}_{2} \mathrm{O}_{2} \mathrm{Cl}$
Gefunden
$\mathrm{Cl} \ldots \ldots \ldots \quad 10 \cdot 09$
$9 \cdot 75$

As. $m$-Xy1o1-azo-4-Chlor-m-Phenylendiamin.

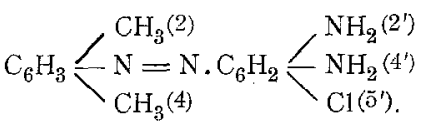

$6 g$ as. $m$-Xylidin wurden mit circa $40 \mathrm{~cm}^{2}$ Wasser versetzt und unter Kühlung $10 \mathrm{~cm}^{3}$ concentrierte Salzsäure zugefügt. Das ausfallende salzsaure $m$-Xylidin gieng bei der nun folgenden Diazotierung mit Natriumnitrit in berechneter Menge wieder in Lösung. Es wurden ungefähr $18 \mathrm{~cm}^{3}$ einer zehnprocentigen Nitritlösung verbraucht. Das Ende der Diazotierung war in üblicher Weise mit Jodkali-Stärkepapier nachzuweisen.

Es wurden außerdem $2 g$ Chlorphenylendiamin in verdünnter Salzsäure gelöst, mit krystallisiertem essigsauren Natron versetzt und mit der hergestellten Diazolösung combiniert. Die Reaction war zu Ende, als eine Probe der Chlorphenylendiaminlösung mit einer kleinen Menge Diazolösung am Filterpapier nicht mehr kuppelte. Der gebildete Azofarbstoff wurde abgesaugt, mit kaltem Wasser nachgewaschen, getrocknet und aus Alkohol umkrystallisiert.

Eigenschaften. Der so erhaltene Farbstoff krystallisiert in schönen rothgelben Nadeln, ist leicht löslich in Alkohol, Äther, Benzol und Chloroform, schwer löslich in kaltem Wasser, viel leichter in heißem. Schmelzpunkt $150^{\circ}$.

Analyse:

$0 \cdot 3369 \mathrm{~g}$ Substanz gaben $0.1764 \mathrm{~g}$ Chlorsilber.

In 100 Theilen:

$$
\mathrm{Cl} \ldots \ldots \ldots \underbrace{\substack{\text { Berechnet für } \\ \mathrm{C}_{14} \mathrm{H}_{15} \mathrm{~N}_{4} \mathrm{Cl}}}_{12 \cdot 89} \quad \frac{\text { Gefunden }}{12 \cdot 93}
$$


In ganz analoger Weise wurden noch folgende Azokörper erhalten:

p-Toluol-azo-4-Chlorphenylendiamin.

$$
\mathrm{C}_{6} \mathrm{H}_{4} \angle \mathrm{N}=\mathrm{N}=\mathrm{C}_{6} \mathrm{H}_{2} \leq \begin{aligned}
& \mathrm{NH}_{2}\left({ }^{\prime}\right) \\
& \mathrm{NH}_{2}\left(4^{\prime}\right) \\
& \mathrm{Cl}\left(5^{\prime}\right) .
\end{aligned}
$$

Die Darstellung dieses Körpers erfolgte genau so wie die des vorstehenden, nur wurde statt $m$-Xylidin $p$-Toluidin diazotiert und mit Chlorphenylendiamin gekuppelt.

Eigenschaften. Der so erhaltene Farbstoff krystallisiert aus Alkohol in schönen orangerothen Blättchen vom Schmelzpunkte $172^{\circ}$. Er ist leicht löslich in Äther, Benzol und Chloroform, schwer löslich in kaltem, leicht in heißem Wasser.

Analyse:

$0 \cdot 4160 \mathrm{~g}$ Substanz gaben $0 \cdot 2252 \mathrm{~g}$ Chlorsilber.

In 100 Theilen:

$$
\mathrm{Cl} \ldots \ldots \ldots \underbrace{\begin{array}{c}
\text { Berechnet für } \\
\mathrm{C}_{13} \mathrm{H}_{13} \mathrm{~N}_{4} \mathrm{Cl}
\end{array}}_{13 \cdot 58} \underbrace{\text { Gefunden }}_{13 \cdot 36}
$$

\section{Benzol-azo-4-Chlor- $m$-Phenylendiamin.}

$$
\mathrm{C}_{6} \mathrm{H}_{5} \mathrm{~N}=\mathrm{N} \cdot \mathrm{C}_{6} \mathrm{H}_{2} \longleftarrow \begin{aligned}
& \mathrm{NH}_{2}\left(2^{\prime}\right) \\
& \mathrm{NH}_{2}\left(4^{\prime}\right) \\
& \mathrm{Cl}\left(5^{\prime}\right)
\end{aligned}
$$

Zur Darstellung wurde Diazobenzolchlorid mit Chlorphenylendiamin gekuppelt.

Eigenschaften. Der so erhaltene Körper ist orangeroth, glänzend und in schönen Blättchen aus Alkohol zu erhalten. Er ist leicht löslich in Alkohol, Äther, Benzol und Chloroform, schwer löslich in kaltem Wasser, leicht in heißem. Schmelzpunkt $151^{\circ}$.

Analyse:

$0 \cdot 3903 g$ Substanz gaben $0 \cdot 2241 \mathrm{~g}$ Chlorsilber.

In 100 Theilen:

$$
\mathrm{Cl} \ldots \ldots \ldots \underbrace{\begin{array}{c}
\text { Berechnet für } \\
\mathrm{C}_{12} \mathrm{H}_{11} \mathrm{~N}_{4} \mathrm{Cl}
\end{array}}_{14 \cdot 35} \underbrace{\text { Gefunden }}_{14 \cdot 12}
$$




\section{Chinonimidderivate.}

Wie zu erwarten war, reagiert das 4-Chlor-m-Phenylendiamin so wie das Phenylendiamin und seine Homologen mit den Nitrosoverbindungen aromatischer tertiärer Amine unter Bildung von Chinonimid-Farbstoffen, von Eurhodinen. Als Beispiel sei die Einwirkung von $p$-Nitroso-Dimethylanilin auf Chlor- $m$-Phenylendiamin angeführt: $7 \cdot 1 \mathrm{~g} 4$-Chlor- $m$-Phenylendiamin wurden in wenig Alkohol gelöst und hierauf wurden $9 \cdot 3 g p$-Nitrosodimethylanilin-Chlorhydrat zugefügt. Es erfolgte intensive Blaufärbung, die nach etwa 20 Minuten langem Erhitzen ins Blauviolette umschlug. Es wurde hierauf mit Wasser verdünnt und dann der Alkohol durch Erhitzen auf dem Wasserbade abgekocht. Durch Aussalzen mit Natriumnitrat schied sich eine schmierige Masse ab, die nach dem Abgießen der überstehenden Flüssigkeit in wenig heißem Wasser gelöst wurde; nach circa 10 Tagen krystallisierte daraus der Farbstoff, der durch nochmaliges Umkrystallisieren aus Alkohol gereinigt wurde. Durch Natronlauge kann man aus Farbstofflösungen die freie Farbbase als braunen Niederschlag fällen, der in Äther mit gelber Farbe löslich ist. Der Bildungsweise nach kommt ihr die nachstehende Formel zu:

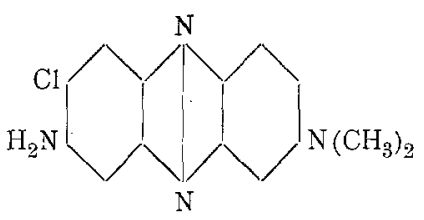

Dimethyldiaminochlorphenazin.

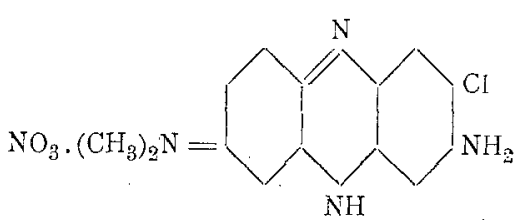

Chinonimidfarbstoff ( $p$-Chinonformel).

Der Farbstoff selbst, das salpetersaure Dimethyldiaminochlorphenazin, besitzt folgende Eigenschaften: er ist leicht löslich in Wasser und Alkohol und ist aus diesen Lösungsmitteln krystallisiert zu erhalten. Durch Einwirkung von concentrierter Schwefelsäure auf die Farbstofflösung erfolgt intensive Blaufärbung, die durch Zusatz von Wasser ins Violette übergeht. Ebenso wirkt concentrirte Salzsäure und nachheriges Verdünnen mit Wasser. Biese Farbenumschläge beruhen darauf, daß die Farbbase ähnlich wie bei anderen Eurhodinen z. B. 
Neutralviolett mit Säuren mehrere Reihen von Salzen bildet, von denen nur die einsäurigen, hier violetten, gegen Wasser beständig sind. Der Farbstoff färbt tannierte und mit Brechweinstein gebeizte Baumwolle schön rothviolett an. Schon $1 / 2 \%$ Ausfärbungen sind verhältnismäßig dunkel.

Wir versuchten nun durch Diazotieren und Behandlung mit Kupferchlorür nach Sandmey er zum 1-2-4-Trichlorbenzol $z \mathrm{u}$ gelangen, das bereits auf anderem Wege hergestellt worden war, ${ }^{1}$ und so auf andere Weise die Constitution des Chlorphenylendiamins festzustellen.

\section{Darstellung von Trichlorbenzol.}

$$
\mathrm{C}_{6} \mathrm{H}_{3}\left\{\begin{array}{l}
\mathrm{Cl}(1) \\
\mathrm{Cl}(2) \\
\mathrm{Cl}(4)
\end{array}\right.
$$

$14 \mathrm{~g}$ Chlor- $m$-Phenylendiamin wurden in concentrierter Salzsäure gelöst und circa $10 \mathrm{~g}$ frisch bereitetes Kupferchlorür zugegeben. Hierauf wurde mit einer Lösung von Natriumnitrit diazotiert. Die Diazotierung wurde derartig durchgeführt, dass man die Nitritlösung vermittels eines Tropftrichters unterhalb des Flüssigkeitsniveaus einfließen ließ, um Gasabsorption $\mathrm{zu}$ erzielen. Auch musste für ausreichende Kühlung gesorgt sein. Das Reactionsproduct wurde mit Wasserdampf überdestilliert, das Trichlorbenzol ausgeäthert, die ätherische Lösung mit Chlorcalcium getrocknet, der Äther verdunstet und der Rückstand fractioniert. Zwischen 208 bis $210^{\circ}$ gieng der Hauptantheil über (Ausbeute $11 \mathrm{~g}$ ).

Eigenschaften. Das so erhaltene Product ist ein schwach gelbliches Öl, von pyridinähnlichem Geruche. Schmelzpunkt $16^{\circ}$ bis $18^{\circ}$, Siedepunkt $207^{\circ}$ bis $208^{\circ}$, bei $213^{\circ}$ corr. In der oben angeführten Literatur wird der Schmelzpunkt mit $16^{\circ}$, der Siedepunkt mit $213^{\circ}$ corr. angegeben.

Die Analyse zeigte, dass der Körper Trichlorbenzol war. Analyse:

$0 \cdot 2421 \mathrm{~g}$ Substanz gaben $0.5715 \mathrm{~g}$ Chlorsilber.

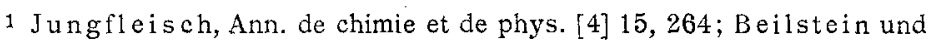
Kurbatow; Ann,, 192, S. 229. 
In 100 Theilen:

$\mathrm{Cl} \ldots \ldots \ldots \underbrace{\begin{array}{c}\text { Berechnet für } \\ \mathrm{C}_{6} \mathrm{H}_{3} \mathrm{Cl}_{3}\end{array}}_{58 \cdot 56} \underbrace{\text { Gefunden }}_{58 \cdot 33}$

Darstellung von 1-Nitro-(2-4-5)-Trichlorbenzol.

$$
\mathrm{C}_{6} \mathrm{H}_{2} \simeq \begin{aligned}
& \mathrm{Cl}(2) \\
& \mathrm{Cl}(4) \\
& \mathrm{Cl}^{(5)} \\
& \mathrm{NO}_{2}(1) .
\end{aligned}
$$

Zur weiteren Identificierung des erhaltenen Trichlorbenzols wurde durch Nitrieren das schon beschriebene 1-Nitro-(2-4-5)Trichlorbenzol dargestellt, ${ }^{1}$ und verfuhren wir folgendermaßen: $5 g$ Trichlorbenzol wurden in einer Schüttelflasche mit ungefähr $10 \mathrm{~cm}^{3}$ concentrierter Salpetersäure (erhalten aus rauchender Salpetersäure durch Erhitzen bis zur Entfärbung) und mit $20 \mathrm{~cm}^{3}$ Schwefelsäure versetzt und etwa 20 Minuten unter Kühlung gut geschüttelt. Die Temperatur des Reactionsgemisches wurde nicht über $30^{\circ}$ bis $35^{\circ}$ steigen gelassen. Sobald sich ein fester Körper abschied, war die Einwirkung vollendet; es wurde das Nitrierungsgemenge in kaltes Wasser eingetragen, das abgeschiedene Nitroproduct abfiltriert, gut nachgewaschen und endlich aus verdünntem Alkohol umkrystallisiert. Das Product war in Ligroin, Äther und Schwefelkohlenstoff leicht löslich. Es zeigte den Schmelzpunkt von $55^{\circ}$. In der Literatur ist für das 1-Nitro-(2-4-5)-Trichlorbenzol der Schmelzpunkt $57^{\circ}$ angegeben. Durch Behandlung des Nitrotrichlorbenzols mit Natronlauge ist es uns gelungen, zu einem Phenol zu gelangen, worüber wir demnächst berichten werden.

Im präparativen Theile der vorliegenden Arbeit wurden wir von Herrn J. Pollak unterstützt, dem wir an dieser Stelle bestens danken.

1 Lesimple, Ann, 137, 123; verg1. Voh1, Zeitschr. für Chemie von Beilstein, Hübner und Fittig, 1867, 122; ferner Beilstein und Kurbatow, Ann., 192, 229 ff. 